\title{
Study of Top Coal Partition and Key Delayed-Action Region for Horizontal Sublevel Top Coal Caving in Deeply Inclined Seam
}

\author{
Jia-fan Zhang $\mathbb{D}^{1},{ }^{1}$ Xiaoyu Liu $\mathbb{D}^{1},{ }^{1}$ Yang Liu, ${ }^{2}$ Xiao-ping Shao $\mathbb{D}^{3},{ }^{3}$ and Hui-mei Zhang ${ }^{1}$ \\ ${ }^{1}$ College of Science, Xi'an University of Science and Technology, Xi'an 710054, China \\ ${ }^{2} X i$ 'an Research Institute of China Coal Technology and Engineering Group Crop, Xi'an 710054, China \\ ${ }^{3}$ College of Energy, Xi'an University of Science and Technology, Xi'an 710054, China \\ Correspondence should be addressed to Xiao-ping Shao; shaoxp_anquan@163.com
}

Received 7 September 2020; Revised 6 April 2021; Accepted 16 April 2021; Published 23 April 2021

Academic Editor: Arunava Majumder

Copyright (c) 2021 Jia-fan Zhang et al. This is an open access article distributed under the Creative Commons Attribution License, which permits unrestricted use, distribution, and reproduction in any medium, provided the original work is properly cited.

Horizontal sublevel caving is the unique way to mine the steep and extra thick coal seams. We aim to determine reasonable height of horizontal subsection, which is crucial to ensure the output and safety of mining. For this aim, a theoretical model is established to determine the theoretical solution of elastic stress distribution in deeply inclined coal seam, and first principal stress expression is derived. And on this basis, related factors affecting the top coal caving property and key delayed-action areas are investigated. The results show that there are four zones in top coal, which are tensile failure zone (TFZ), tensile damage zone (TDZ), elastic zone (EZ), and compression-shear damage zone (CSDZ). The elevation of sublevel height cannot increase the release of top coal. It is necessary to determine the key region of affecting the top coal caving. The key delayed-action region (KDAR) consists of EZ and TFZ in top coal; the EZ and TFZ are type I and II KDAR, respectively, and type I is the key area of weakening top coal. In order to effectively weaken KDAR, opening coal channel of core region, and especially effectively obstructing the continuous moving upward of top coal caving arch structure with the help of weakening top coal body in type I KDAR, would promote caving property of top coal and improve the working face extraction rate.

\section{Introduction}

The energy industry has a great influence on the development of the society [1-3]. Steeply inclined coal seams occur widely in many coal-producing areas, including China, Spain, and France [4]. Deeply inclined coal seam has various kinds of occurrence conditions. Coal seam dip angle is from $45^{\circ}$ to $90^{\circ}$, and the roadway layout, increment of production, and mining safety of working face have many problems to be solved [5-8]. However, traditional mining methods, such as slicing mining, storage mining, middle-deep hole blasting, and top coal mining with sliding top coal beam [9], cannot solve these problems reasonably. Horizontal section top coal caving as a very safe and effective mining method had been widely used recent years. This method uses the horizontal thickness of coal seam to put the fully mechanized mining face. But the length of deeply inclined seam sublevel top coal caving's working face is relatively small, regularly $10 \mathrm{~m}$ to
$50 \mathrm{~m}$. Thus, in order to increase the working productivity, engineers must increase the sublevel height of sublevel top coal caving working face; that means to make the caving height. Based on $115^{\text {th }}$ of the Coalmine Safe Protocol, the percentage of horizontal section top coal caving method of deeply inclined coal seam mining area should be less than $12.5 \%$. Recently, the sublevel height of working face in Jiangou coal mine at Wulumuqi, Xinjiang province (coal seam dip angle $87^{\circ}$ ), is $28 \mathrm{~m}$, and the sublevel height of working face in Tiechanggou coal mine is already $18 \mathrm{~m}$. With the increment of sublevel height and the difference of coal seam dip angle, the deformation, disruption, and caving property of the top coal will change significantly.

Partition of top coal to determine the influence dip angle and sublevel height on partition of top coal is analysed by Shi and Gao with deflection function [10]. Zhang thought the destructive process of top coal could be divided into complete area, disruption development area, fracture 
development area, and crushing area [11]. Jin et al. believed that the deformation of top coal after mining and loading support pressure of front coal wall could be clearly separated to prominent deformation zone, top coal fracturing zone, top coal relaxation zone, and top coal caving zone [12]. Shi separated top coal along the direction of roof-control zone to shear crack zone (front zone) and compressive and tensile failure zone [13]. For the steep coal seams, high section mining would critically increase the sublevel height [14-17]. The method only relied on compression of mine and effect of support would not make sure the top coal can be effectively mining $[18,19]$. In this case, the supportive weakening of top coal like blasting or hydrofracturing should be used. Before that, the key zone of top coal weakening should be found. Liao et al. investigated the overburden failure law of fully mechanized face based on deflection equation of overlying strata beam [20]. Taking a cohesive element and considering the influence of geologic bedding plane around the roadway, the deformation and corresponding improved support scheme were analysed by $\mathrm{Wu}$ et al. [21]. Numerical simulation was carried out by Qi et al. to analyse the deformation and failure characteristics of surrounding rock in steep seam with weak structural plane [22].

The above research methods and results have important implications for understanding the deformation and failure of deeply inclined seam, but the stress distribution of top coal was lacking, which led to partition and KDAR of top coal not being quickly and properly determined by engineers. In this study, elastic solution of stress distribution is analysed based on top coal mechanical model, and first principal stress expression is derived. On this basis, related factors affecting the top coal caving property and key delayed-action areas are investigated to promote caving property of top coal and improve the working face extraction rate. Moreover, contributions of existing studies and this paper are elaborated in Table 1.

\section{Establishment and Calculation of Top Coal Mechanical Model}

2.1. Establishment of Top Coal Mechanical Model. The mining of top coal is a dynamic process. During that process, the shape and stress state of top coal changed all the time. For steep coal seam, the most dangerous situation of top coal mining is that when the coal breaking effect by support is finished, the stable top coal structure is formed in upper part of top coal, which will generate separation between the loose coal and upper top coal. In this case, the loose coal could be mined by support caving window, while the upper top coal could not be mined. In the circumstances, the upper top coal will keep weak relationship with coal wall side body. Or the upper top coal has no relationship with it, which means the side top coal at roof area is suspended in midair, and the coal roof and coal floor have some certain constraint, which formed the parallelogram prism hanging in the air. This is the most dangerous caving structure of top coal. The solution of this mechanics problem is a space problem of elasticity, whose calculation is so complicated.
In order to simplify calculation, researchers simplify the problem to a plane stress problem along the direction of dip angle. When top coal roof is stable, considering the coal seam dip angle is bigger than $45^{\circ}$, the normal component of load pass to the top coal in the coal roof is small. Therefore, parallelogram top coal mechanical model which has fixed support at the left and right edges and uniform load at top edge and free edge at the bottom can be built, as shown in Figure 1. Researchers set the coal seam dip angle to $\alpha$. Along horizontal direction of coal seam, working face length and slope height are $2 a$ and $2 b$, respectively. The uniform load intensity of top coal is $q$.

At the left and right boundary of the model, displacement boundary condition can be simplified to

$$
w=0, \quad \frac{\partial w}{\partial x}=0 \quad y=(\sin \alpha) x \pm a .
$$

At the top and bottom boundary of model, stress boundary condition can be simplified to

$$
\begin{aligned}
& \sigma_{y}=-q, \quad \tau_{x y}=0, \quad y=b \sin \alpha, \\
& \sigma_{y}=0, \quad \tau_{x y}=0, \quad y=-b \sin \alpha .
\end{aligned}
$$

As shown in Figure 1, this mechanical model involves the selection of stress function for parallelogram thin plate. Parallelogram plate is the special case of skew plate, which belongs to irregular plates in plate mechanics. Due to its complex shape, classical solution is very difficult. Thus, researchers used stress variation method to find its analytic solution. In this case, researchers turn boundary value problem in elastic mechanics to functional stationary value problem.

2.2. Selection and Calculation of Stress Function. The stress in top coal should satisfy the equilibrium differential equation, stress boundary condition, and compatibility equation and assume that the body force does not change, while stress has very tiny changes $\delta \sigma_{x}, \delta \sigma_{y}$, and $\delta \tau_{x y}$, which is called virtual stress or variation of stress. Therefore, in certain boundaries (surface force is not given), variation of stress component will follow the variation of surface force, which is $\delta X, \delta Y$, and $\delta Z$, and the variation of deformation potential energy, which is $\delta U$. The variation of deformation potential energy is also equal to the work done by the variation of the surface force on the actual displacement. Thus, the stress variation equation is as follows:

$$
\delta U=\iint(u \delta X+v \delta Y+w \delta Z) d S .
$$

The model of steep coal seam top coal structure is shown in Figure 1, which can be considered as plane stress problem. Taking the unit thickness in the $z$ direction, the deformation potential energy of the coal body is

$$
U=\frac{1}{2 E} \iint\left[\sigma_{x}^{2}+\sigma_{y}^{2}-2 \mu \sigma_{x} \sigma_{y}+2(1+\mu) \tau_{x y}^{2}\right] d x d y .
$$

Considering the top coal is deformation body and stress boundary conditions, $\sigma_{x}, \sigma_{y}$, and $\tau_{x y}$, should be independent 
TABLE 1: Contributions of existing studies and this paper.

\begin{tabular}{|c|c|c|c|c|c|c|}
\hline Author (s) & Analytical method & Dip angle & Sublevel height & Working face length & Partition & KDAR \\
\hline Shi and Gao [10] & Deflection & Consideration & Consideration & $\mathrm{NC}$ & Analysis & NA \\
\hline Zhang [11] & Deflection & NC & Consideration & $\mathrm{NC}$ & Analysis & NA \\
\hline Jin et al. [12] & Deflection & Consideration & Consideration & Consideration & Analysis & NA \\
\hline Shi $[13]$ & Deflection & NC & Consideration & Consideration & NA & NA \\
\hline Qi et al. [22] & Modelling & NC & NC & $\mathrm{NC}$ & NA & NA \\
\hline Liao et al. [20] & Deflection & Consideration & Consideration & Consideration & NA & NA \\
\hline Wu et al. [21] & Modelling & NC & $\mathrm{NC}$ & $\mathrm{NC}$ & NA & NA \\
\hline This paper & Stress & Consideration & Consideration & Consideration & Analysis & Analysis \\
\hline
\end{tabular}

NC: no consideration; NA: no analysis.

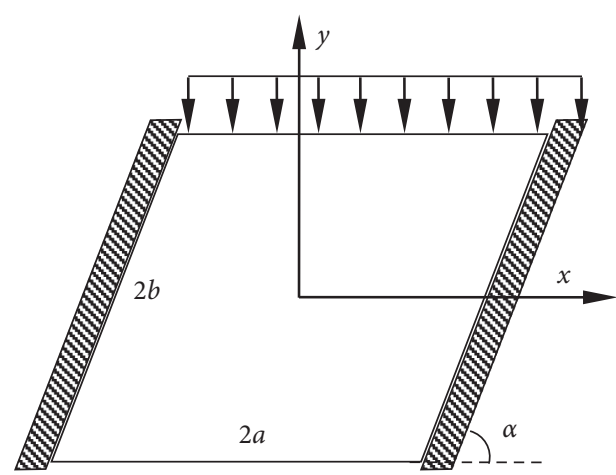

Figure 1: Mechanics model of top coal structure for deeply inclined seam.

of elastic constants, so the potential energy can be simplified to

$$
U=\frac{1}{2 E} \iint\left[\sigma_{x}^{2}+\sigma_{y}^{2}+2 \tau_{x y}^{2}\right] d x d y
$$

In plane problem, if body force component is constant, there will be stress function, whose stress components $\sigma_{\mathrm{x}}, \sigma_{\mathrm{y}}$, and $\tau_{\mathrm{xy}}$ can be represented by stress function $\varphi$ :

$$
\sigma_{x}=\frac{\partial^{2} \phi}{\partial y^{2}}-X x, \quad \sigma_{y}=\frac{\partial^{2} \phi}{\partial x^{2}}-Y y, \quad \tau_{x y}=-\frac{\partial^{2} \phi}{\partial y \partial x} .
$$

From formulas (5) and (6), the deformation potential energy expression represented by stress function is as follows:

$$
U=\frac{1}{2 E} \iint\left[\left(\frac{\partial^{2} \phi}{\partial y^{2}}-X x\right)^{2}+\left(\frac{\partial^{2} \phi}{\partial x^{2}}-Y y\right)^{2}+2\left(\frac{\partial^{2} \phi}{\partial y \partial x}\right)^{2}\right] d x d y .
$$

Considering the stress and displacement boundary conditions of coal body, take stress variation equation as follows:

$$
\frac{\partial U}{\partial A_{m}}=0
$$

Formula (7) can transpose to the following:

$$
\begin{aligned}
& \iint\left[\left(\frac{\partial^{2} \phi}{\partial y^{2}}-X x\right) \frac{\partial}{\partial A_{m}}\left(\frac{\partial^{2} \phi}{\partial y^{2}}\right)+\left(\frac{\partial^{2} \phi}{\partial x^{2}}-Y y\right) \frac{\partial}{\partial A_{m}}\left(\frac{\partial^{2} \phi}{\partial x^{2}}\right)+2\left(\frac{\partial^{2} \phi}{\partial y \partial x}\right) \frac{\partial}{\partial A_{m}}\left(\frac{\partial^{2} \phi}{\partial y \partial x}\right)\right] d x d y=0 . \\
& \text { (9) can be used to get the undetermined co- } \\
& \text { f the stress function is correct. When applying } \\
& \text { iation method, we can set the stress function } \varphi
\end{aligned}
$$
efficient $A_{m}$ if the stress function is correct. When applying the stress variation method, we can set the stress function $\varphi$ as follows:

$$
\varphi=\varphi_{0}+\sum_{m} A_{m} \varphi_{m}
$$

In formula (10), $A_{1}, A_{2} \ldots A_{m}$ are independent coefficients.

Based on the boundary shape and stress conditions of top coal, after many times of trying and correcting, the researchers find the biharmonic function shown in formula (10) as stress function, which also satisfied boundary conditions. Finally, the stress function is as follows:
From formulas (10) and (11), the undetermined coefficients of stress function are as follows:

$$
A_{1}=-\frac{1}{24 \pi b^{3} \sin \alpha}\left[\sin \left(\frac{\pi}{\sin \alpha}\right) \sin \alpha-\cos \left(\frac{\pi}{\sin \alpha}\right)\right], \quad A_{2}=0 .
$$

From formulas (6), (11), and (12), $\sigma_{x}, \sigma_{y}$, and $\tau_{x y}$ are determined as follows: 


$$
\begin{aligned}
\sigma_{x} & =\frac{\pi q x^{2}}{4 b^{2} \sin ^{2} \alpha} \sin \left(\frac{\pi y}{b \sin \alpha}\right)+6 A_{1} q a^{2} y, \\
\sigma_{y} & =-\frac{1}{2} q\left(1+\frac{y}{b \sin \alpha}\right)-\frac{1}{2 \pi} q \sin \left(\frac{\pi y}{b \sin \alpha}\right), \\
\tau_{x y} & =-\frac{q x}{2 b \sin \alpha}\left[1+\cos \left(\frac{\pi y}{b \sin \alpha}\right)\right]
\end{aligned}
$$

2.3. Failure Criterion of Top Coal. The coal body is typical brittle material, which has low tensile strength, middle shear strength, and high compressive strength. Thus, the maximum tension-stress theory should be used as criterion of top coal's failure, and then the researchers determined that the maximum tensile stress is the main factor causing the instability of the lower top coal, which means if only the maximum tensile stress reaches the tensile strength of the top coal, the lower top coal will be breaking and caving. The expression is as follows:

$$
\sigma_{1} \geq t
$$

In the formula, $\sigma_{1}$ is maximum principal stress and $\sigma_{t}$ is the tensile strength limit of coal body.

After the analysis of top coal stress state, the researchers get the first principal stress of top coal:

$$
\sigma_{1}=\frac{1}{2}\left[\left(\sigma_{x}+\sigma_{y}\right)+\sqrt{\left(\sigma_{x}-\sigma_{y}\right)^{2}+4 \tau_{x y}^{2}}\right] .
$$

Substituting formula (13) into (15) and setting the half of sublevel height $0.5 h=b \sin \alpha$, the researchers got the first principal stress of top coal as

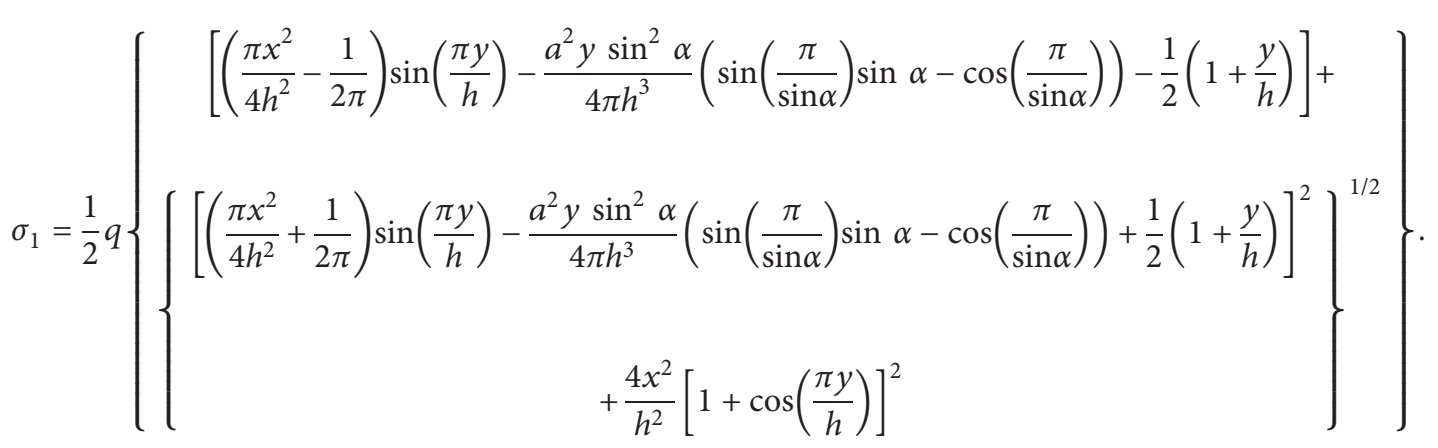

Formula (16) is the first principal stress expression at any point of the parallelogram top coal body. The analysis of formula (16) shows that the first principal stress in the top coal is related to the load intensity $q$, coal seam dip angle $\alpha$, sublevel height $h$, and the working face length $2 a$, in which only the load intensity $q$ is linear with the first principal stress, and the rest are nonlinear relations with it. It should be noted that the value of the first principal stress is related to the position coordinates in the top coal body. From the perspective of top coal caving, the researchers should pay attention to the falling height of the top coal. Therefore, the researchers choose the point which is $5 \mathrm{~m}$ with the lower boundary of top coal from $y$ axis to study the influence of the relevant factors on the first principal stress.

\section{Analysis of Relevant Factors of Top Coal Breaking}

3.1. Load Intensity. The analysis of formula (16) shows that there is a linear relationship between the first principal stress $\sigma 1$ and the load intensity $q$ at any point in top coal body. According to the first theory of strength, when $\sigma 1$ exceeds the uniaxial tensile strength of the coal body, the top coal body will break. Thus, increasing the load intensity $q$ of the top coal body will have a good effect on top coal caving. In actual engineering, the applied body of the load intensity $q$ is a rock layer which is already falling above the top coal and a small part of the remaining coal, and these two parts' increasing effect on the amplitude of load intensity $q$ is small. Therefore, in the case where sublevel height is greatly improved, the assistant way to crush the key area of the top coal should be considered as a feasible method.

3.2. Coal Seam Dip Angle. Figure 2 shows the relationship between the value of first principal stress $\sigma 1$ and the coal seam dip angle when the coal seam dip angle changes from $45^{\circ}$ to $90^{\circ}$. The analysis shows that when the coal seam dip angle is between $45^{\circ}$ and $55^{\circ}, \sigma_{1}$ is increased rapidly in a way of near straight line with a large slope; when the coal seam dip angle is between $55^{\circ}$ and $75^{\circ}$, the increment of $\sigma 1$ becomes much slower, whose slope generally approaches the 0 ; when the coal seam dip angle is between $75^{\circ}$ and $90^{\circ}, \sigma 1$ has tended to a constant value, presenting a nearly horizontal line with a slope of nearly 0 . The general trend of the curve is that $\sigma_{1}$ tends to a constant value beyond a certain dip angle, which means that, for a certain coal strength, the general trend of top coal falling height is increasing with the increase of coal seam dip angle. That is, the top coal caving characteristics are increasing, but when coal seam dip angle increases to $75^{\circ}$, it will be generally stable. Moreover, this effect has obvious interval feature; when the coal seam dip angle is between $45^{\circ}$ and $55^{\circ}$, the increment effect of top coal caving characteristics is most obvious. 


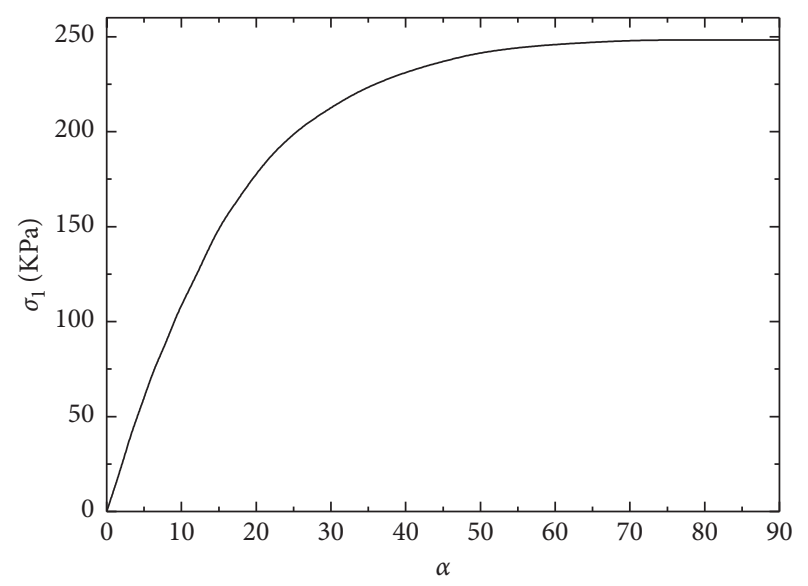

FIgURE 2: The relation curve between $\sigma_{1}$ and coal seam inclination $\alpha$.

3.3. Sublevel Height. The relationship between first principal stress $\sigma_{1}$ and sublevel height is shown in Figure 3. Choose the sublevel height from $16 \mathrm{~m}$ to $32 \mathrm{~m}$. The picture shows that, with the increment of sublevel height, $\sigma_{1}$ decreases in form of near straight line rapidly. It shows that, for certain coal strength, the top coal caving characteristics decrease with the increase of sublevel height. When the sublevel height is $16 \mathrm{~m}$, the coal with tensile strength of $0.28 \mathrm{MPa}$ can be caved at the position of $5 \mathrm{~m}$ below the top coal body. When the sublevel height is increased to $32 \mathrm{~m}$, only the coal with tensile strength of $0.07 \mathrm{MPa}$ can be caved at the same point. Therefore, the perspective of top coal caving characteristics is unfavourable to increase the sublevel height for the caving of top coal.

3.4. Working Face Length. Figure 4 shows the curve of the first principal stress $\sigma_{1}$ when working face length is changed from $10 \mathrm{~m}$ to $50 \mathrm{~m}$. It shows that, with the increase of working face length, $\sigma_{1}$ increases gradually in the form of nonlinearity, which means that, for a certain coal strength, the caving characteristics of top coal increase with the increment of the working face length. When the working face length is $20 \mathrm{~m}$, only the coal with tensile strength of $0.12 \mathrm{MPa}$ can be caved at the position of $5 \mathrm{~m}$ below the top coal body. When the working face length is increased to $50 \mathrm{~m}$, the coal with tensile strength of $0.76 \mathrm{MPa}$ can be caved at the same point. Therefore, in the deeply inclined seam sublevel top coal caving, in order to promote the top coal caving effect, for a single coal seam, we should make full use of the horizontal thickness of coal seam to increase the working face length. For the deeply inclined close seam, the combined arrangement should be used to increase the working face length.

\section{Determination of Top Coal Partition and Key Delayed-Action Region}

4.1. Top Coal Partition. The above analysis shows that, for the certain deeply inclined seam, horizontal sublevel top coal caving is adopted. In the case that coal seam dip angle, the load intensity, and the working face length are fixed, the sublevel height is the key factor to the top coal caving. In actual engineering, if we want to increase the production by increasing the sublevel height, we must use an assisting way to weaken the top coal. But first of all, we should theoretically analyse the stress distribution characteristics of the top coal body corresponding to the variation of the height sublevel height, especially to reveal the key delayed-action region (KDAR), which has effect on the top coal caving. We should do scientific zoning of the most unfavourable caving state of the top coal body of the deeply inclined seam, which can provide theoretical basis for the implementation of the weakening technology in the KDAR of the top coal caving.

Considering formula (17) and taking the deeply inclined seam with the dip angle of $68^{\circ}$ as an example, set the working face length to $28 \mathrm{~m}$ and the sublevel height to $20 \mathrm{~m}$; we can get the contour plot of $\sigma_{1}$ in the top coal, which is shown in Figure 5. When $\sigma_{1}$ in the top coal is bigger than its tensile strength $\sigma_{t}$ and it has free space for the top coal to fall, the falling will happen. This zone is defined as the tensile failure zone (TFZ). When $\sigma_{1}$ is between the tensile strength $\sigma_{t}$ and 0 , it is considered that the coal is in a tensile damage state, but no damage occurs. It can be defined as the tensile damage zone (TDZ). When $\sigma_{1}$ is between 0 and the elastic compressive limit $\sigma_{\mathrm{e}}$, it is considered that the coal is in elastic state, and it can be defined as an elastic zone (EZ). When $\sigma_{1}$ is bigger than the elastic compressive limit $\sigma_{\mathrm{e}}$, the top coal is in the compression-shear damage developing state. At this state, the top coal may have the compression-shear failure, so it can be defined as the compression-shear damage zone (CSDZ). Therefore, according to the first theory of strength, the top coal can be divided into four zones according to the different range of $\sigma_{1}$. From the bottom to the top, the top coal can be divided into TFZ, TDZ, EZ, and CSDZ. When the occurrence conditions of coal seam, mining technical parameters, and the tensile and compressive strength of coal body are changed, the range of each subdivision will also change.

\subsection{The Influence of Sublevel Height on Top Coal Partition.} Increasing the sublevel height to increase coal production per unit push degree is the main technological method to develop deeply inclined seam caving. The research about the influence of the change of sublevel height on the zoning of top coal can lay a theoretical foundation for engineering application. Figure 6 shows the top coal subdivision of different sublevel height, which is $15 \mathrm{~m}, 25 \mathrm{~m}$, and $35 \mathrm{~m}$, respectively.

For the TFZ, the height of caving arch is the key factor to determine whether the top coal body can fall freely without external force. Figure 6 shows that when the sublevel height is $15 \mathrm{~m}$, the height of the caving arch is about $3.9 \mathrm{~m}$. Then it decreased step by step. When the sublevel height is $35 \mathrm{~m}$, the height of the caving arch is only $2.97 \mathrm{~m}$. It shows that the height of the caving arch decreases with the increase of the sublevel height. That indicates that the bigger the sublevel height, the greater the strength of assistant methods to weaken the top coal. Meanwhile, with the increase of 


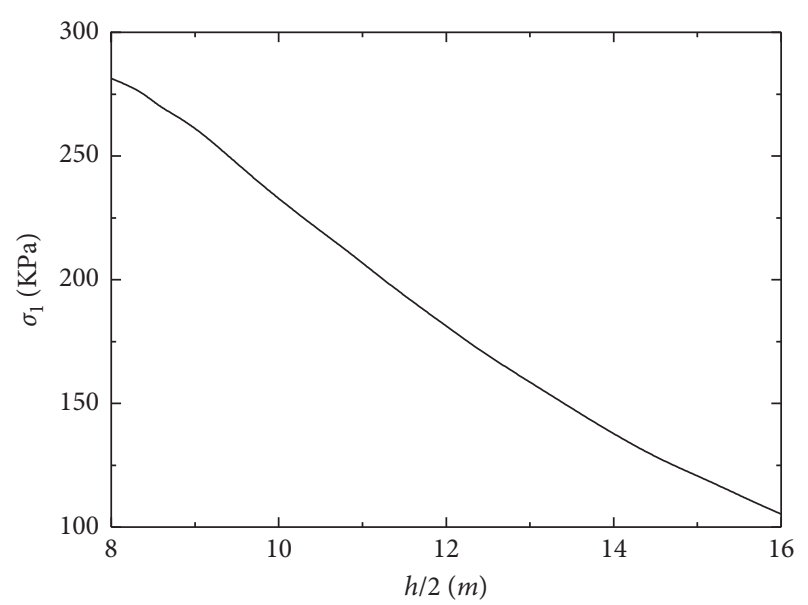

Figure 3: The relation curve between $\sigma_{1}$ and sublevel height $h / 2$.

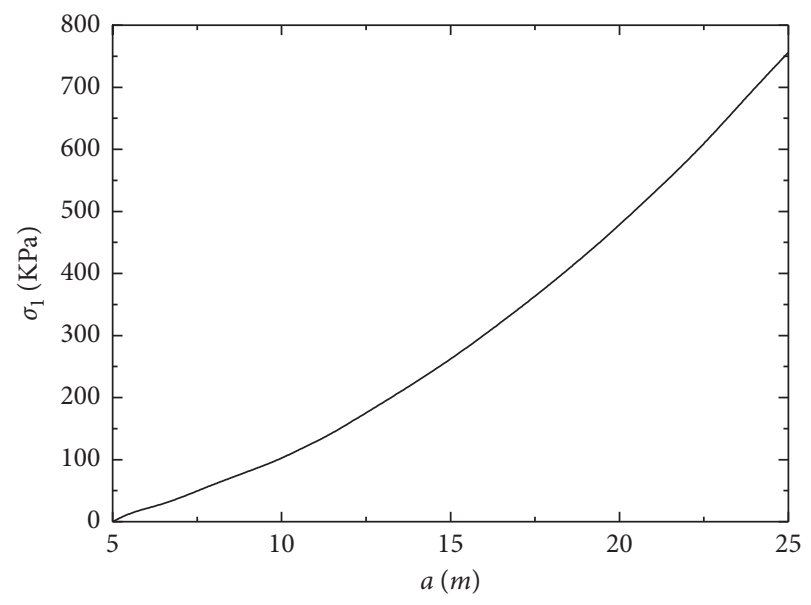

FIgURE 4: The relation curve between $\sigma_{1}$ and the working face length $a$.

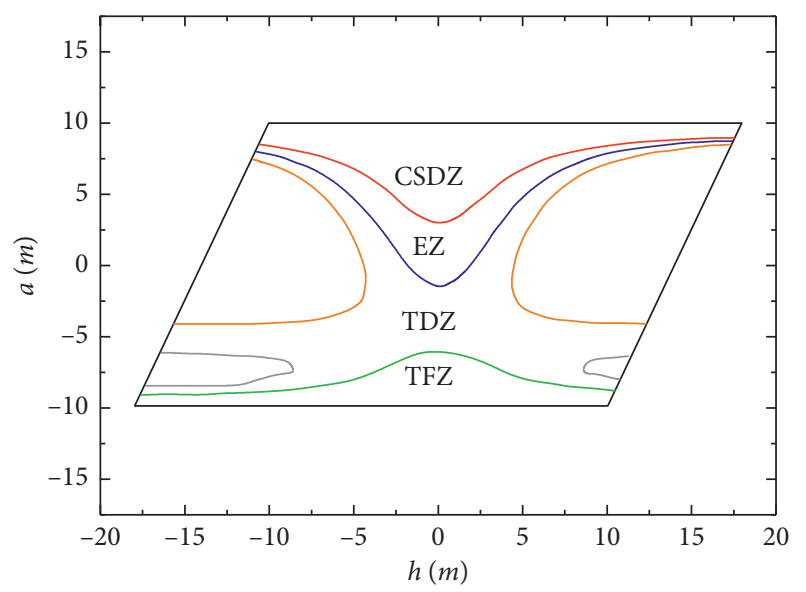

FIGURE 5: The top coal partition along inclination in deeply inclined seam.

sublevel height, TDZ and EZ develop over top coal geometry centre along the vertical direction. The original EZ of the floor side decreases gradually with the increase of sublevel height, and the change of the roof side is little. The height of the upper compression-shear damage zone increases with the increase of sublevel height, from when sublevel height is $15 \mathrm{~m}$ and its height is $6.4 \mathrm{~m}$ to when sublevel height is $35 \mathrm{~m}$ and its height is $11.5 \mathrm{~m}$.

4.3. The Determination of KDAR. The cracking of top coal body in deeply inclined seam is the result of the damage accumulation and along the trend and structure failure and instability along incline. In order to increase the caving rate of top coal, the mine pressure should be rationally used along the trend to increase the damage accumulation of top coal, and the self-stable caving structure in the coal body should be broken along the incline [23-25]. The specific process of the top coal caving along incline is that the caving first begins in the TFZ of the lower coal body. With the falling and caving of the coal body in the TFZ, the mechanical structure of the top coal changes and the stress state evolves and develops until the first principal stress becomes nearly zero in TDZ. When the third principal stress is completely compressive, the top coal caving falling will stop and it forms a self-stable caving arch. The upper coal is blocked by this structure; that is, the top coal has a KDAR during the caving process. Considering that the TDZ might form a temporary stable caving arch structure during the development of top coal caving, the damage accumulation in the EZ is smaller and the coal body is not caved effectively. So, as shown in Figure 7, the KDAR can be determined as the sum of EZ and TDZ. We determine that the elastic area is class I KDAR and the TDZ is class II KDAR. The key of coal body weakening is to destroy the temporary stable caving arch structure [26-28]; and one important aspect of coal body weakening of class I KDAR is forming the barrier zone of the falling arch structure and the use of the separated body force after coal body weakening on TDZ, effectively destroying temporary stable falling arch structure. The other aspect is to make compression-shear damage zone lose its lower support and destroy the falling arch by upper load. Therefore, the class I KDAR is significant to coal body weakening, and the class II KDAR should be applied to assistant method of coal body weakening to cooperate with the work done to class I.

To sum up, we should study KDAR, open the coal caving channel in the core area, and effectively destroy the selfstable falling arch of the top coal. These methods will help the damage development of the coal body above the arch and then reduce the coal loss and increase the productivity of the working face. The determination of KDAR provides a scientific basis for the coal body weakening method in deeply inclined seam sublevel top coal caving.

\section{Engineering Application}

One mine belonging to Shenhua Xinjiang branch company has coal seam dip angle of $68^{\circ}$, horizontal thickness of $28.6 \mathrm{~m}, \mathrm{E} 2 \mathrm{E}+592 \mathrm{~B}_{1+2}$ sublevel caving working face having sublevel height of $20 \mathrm{~m}$, working face length of $565 \mathrm{~m}$, and design production rate of working face being $73 \%$. In order 


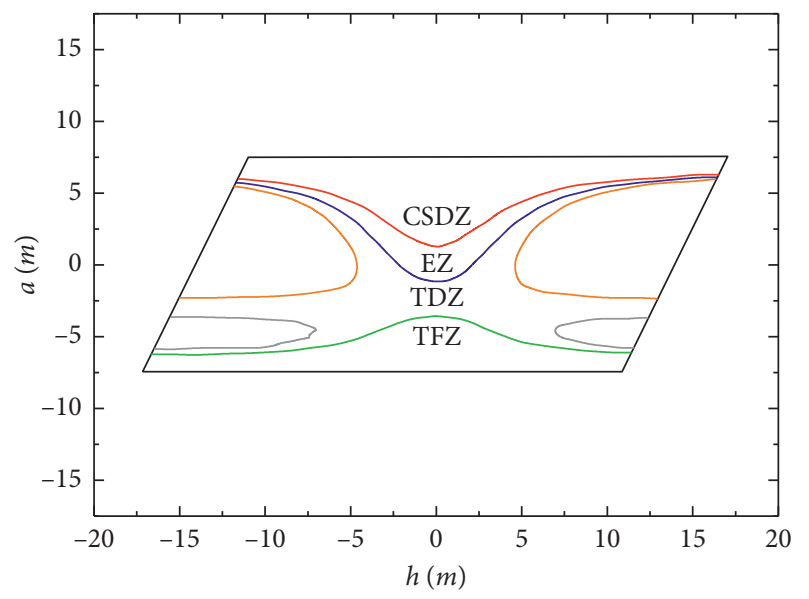

(a)

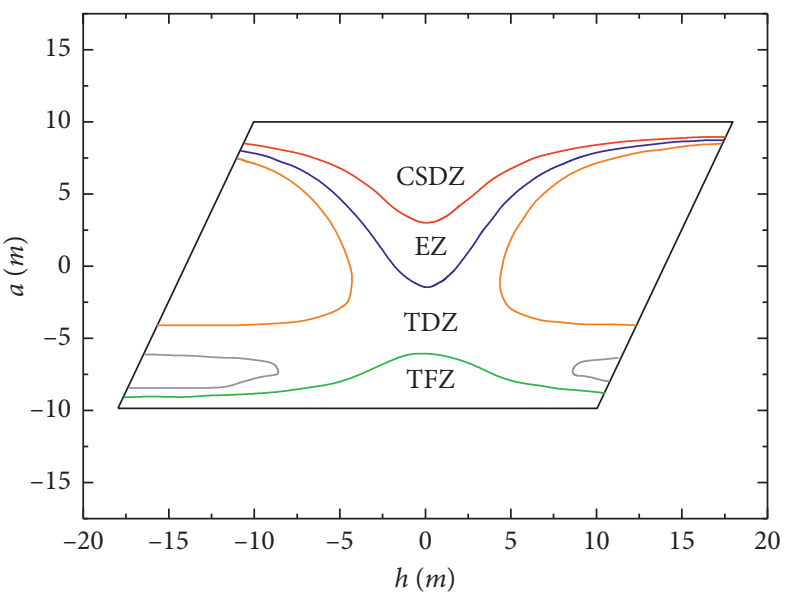

(b)

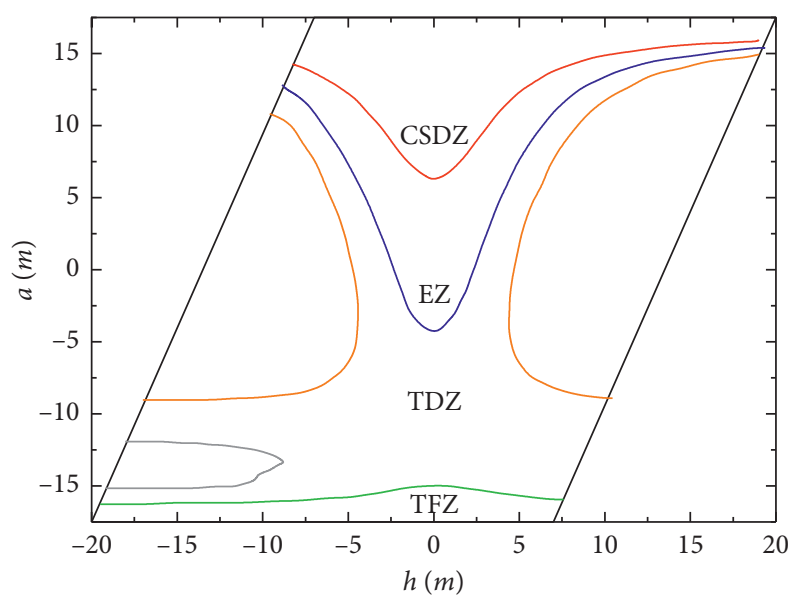

(c)

Figure 6: The top coal partition map of (a) $h=15$; (b) $h=25$; and (c) $h=35$.

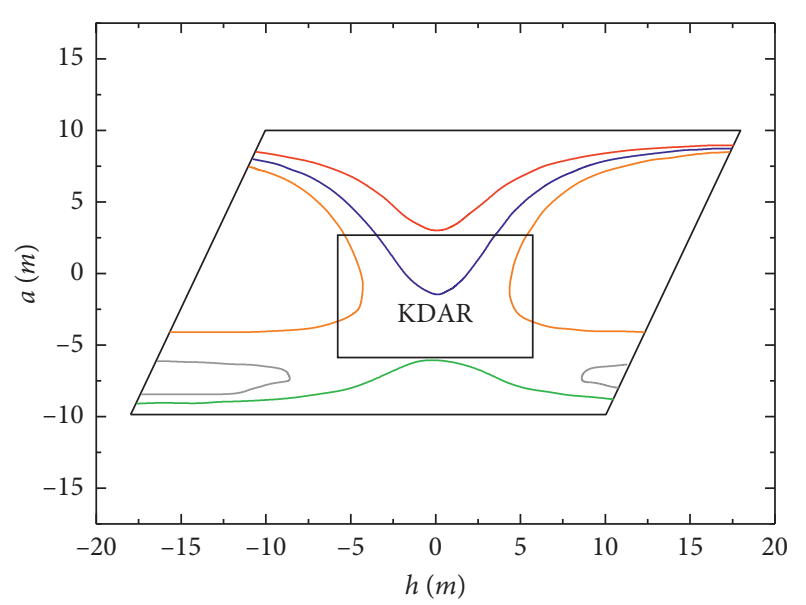

Figure 7: The KDAR in top coal body.

to analyse the caving effect of KDAR, as shown in Figure 8, single hole and fan-shaped hole arrangement is used, while the single hole is arranged as the traditional way and the fan hole arrangement is aimed at the weakening of the coal body in the KDAR. The drilling angle of the single hole is $32^{\circ}$, the length of the drilling hole is $19 \mathrm{~m}$, the angle of the fan-shaped hole is $30^{\circ}, 50^{\circ}$, and $70^{\circ}$, respectively, whose drilling lengths are $15 \mathrm{~m}, 15 \mathrm{~m}$, and $10 \mathrm{~m}$, and the fracturing water pressure of the coal seam is $8-12 \mathrm{MPa}$.

The water injection experiment is divided into two stages. During June 22nd to July 13th, use the single hole zone; the average mining rate was $72.80 \%$. During July 14 th to September 21st, use the fan-shaped hole zone; the average mining rate was $87.50 \%$, which was $14.7 \%$ higher than that of the single hole. Comparing the two different stages of water injection experiment, as shown in Table 2, the average daily productivity of single hole area and fan-shaped hole area is $1510.2 \mathrm{t}$ and $2046.7 \mathrm{t}$, respectively. The average daily mining rate of fan hole is $35.5 \%$ higher than that of the single hole, indicating that the effect of fan-shaped hole injection to weaken the top coal is better than the single row hole. This method effectively opens the coal caving tunnel in the core area, and it effectively obstructs the continuous upward movement of the falling arch structure, so that the ideal top coal caving effect is achieved. 


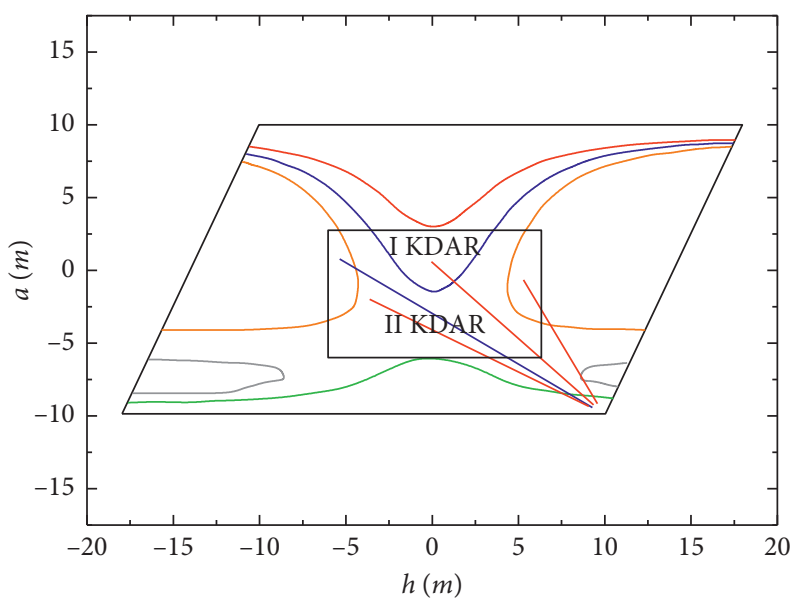

FIGURE 8: Weakening scheme in KDAR; blue and red line are single hole and fan-shaped hole arrangement, respectively.

TABLE 2: Weakening top coal using water injection method and production condition at working face.

\begin{tabular}{lcccc}
\hline Time & Average advance distance $(\mathrm{m})$ & Average advance time $(\mathrm{d})$ & Average daily productivity $(\mathrm{T})$ & Average productivity rate $(\%)$ \\
\hline $5.16-6.21$ & 0.0 & 0.0 & 1753.0 & 71.17 \\
$6.22-7.31$ & 28.4 & 10.8 & 1613.0 & 78.50 \\
$8.1-8.31$ & 15.1 & 4.5 & 1900.6 & 82.10 \\
$9.1-9.21$ & 34.8 & 8.4 & 2526.1 & 95.80 \\
$9.22-10.12$ & 0.0 & 0.0 & 1716.2 & 72.40 \\
\hline
\end{tabular}

\section{Conclusions}

(1) By establishing parallelogram mechanical model of the top coal body in deeply inclined seam, this paper is the first to give the theoretical solution of elastic stress distribution with stress function method. On this basis, the first principal stress expression at any point in the top coal is derived and the maximum tensile stress is determined as the main factor causing the destabilization of the lower top coal. The result also suggests that increasing sublevel height is unfavourable to the caving of top coal, which implies that increasing the productivity can only be achieved by increasing working face.

(2) The study determines that the elastic zone (EZ) and the tensile damage zone (TDZ) are the key delayedaction region (KDAR) in the top coal, the EZ is the class I KDAR, the TDZ is the class II KDAR, and the class I KDAR is the key area of the coal body weakening. The engineering practice shows that the effective weakening of coal body in the KDAR, the opening of coal caving channel in the core area, and the effective obstruction of falling arch structure's continuous upward movement are very useful for improving the mining rate of the working face.

(3) The cracking of the top coal body is the result of damage accumulation under complex stress condition in deeply inclined seam, and stress-strain curve displays the nonlinear characteristics in this region. Therefore, theoretical solution of elastic stress distribution is extended by considering damage and plasticity to describe its nonlinear deformation
[21, 29]. This model can also be extended by combining with strength criterion of complex stress condition to predict its different failure mechanism $[30,31]$. Moreover, in order to make a comprehensively understandable mechanism of progressive failure of deeply inclined seam, further research is required to determine stress distribution after roof breakage [20].

\section{Abbreviations}

$w: \quad$ Vertical displacement

$\sigma_{y}, \sigma_{\mathrm{x}}: \quad X$ and $Y$ direction stresses

$\sigma_{x y}: \quad X-Y$ plane shear stress

$\alpha: \quad$ Coal seam dip angle

$U$ : Deformation potential energy

E: $\quad$ Elasticity modulus

$a, b$ : Working face length and slope height

$q$ : Uniform load intensity of top coal

$\varphi: \quad$ Stress function

$\sigma_{1}: \quad$ First principal stress

$\sigma_{t}: \quad$ Tensile strength

$h$ : $\quad$ Sublevel height

KDAR: Key delayed-action region

CSDZ: Compression-shear damage zone

TDZ: Tensile damage zone

EZ: $\quad$ Elastic zone

TFZ: Tensile failure zone.

\section{Data Availability}

The data used to support the findings of this study are included within the article. 


\section{Conflicts of Interest}

The authors declare that they have no conflicts of interest.

\section{Acknowledgments}

This study was supported by the Natural Science Foundation of Shaanxi Province (2020JZ-53), Natural Science Foundation of Shaanxi Provincial Department of Education (19JK0521), Foundation of Xi'an University Science and Technology (2018QDJ030), and National Natural Science Foundation of China (11172232).

\section{References}

[1] M. Sarkar and B. Sarkar, "How does an industry reduce waste and consumed energy within a multi-stage smart sustainable biofuel production system?" Journal of Cleaner Production, vol. 262, Article ID 121200, 2020.

[2] J. Jemai, B. D. Chung, and B. Sarkar, "Environmental effect for a complex green supply-chain management to control waste: a sustainable approach," Journal of Cleaner Production, vol. 277, Article ID 122919, 2020.

[3] B. K. Dey, S. Pareek, M. Tayyab, and B. Sarkar, "Autonomation policy to control work-in-process inventory in a smart production system," International Journal of Production Research, vol. 59, no. 4, pp. 1258-1280, 2021.

[4] H. Shengquan, S. Dazhao, H. Xueqiu et al., "Coupled mechanism of compression and prying-induced rock burst in steeply inclined coal seams and principles for its prevention," Tunnelling and Underground Space Technology, vol. 98, Article ID 103327, 2020.

[5] Y. Ru, X. Lv, J. Guo et al., "Real-Time prediction model of coal and gas outburst," Mathematical Problems in Engineering, vol. 2020, Article ID 2432806, 5 pages, 2020.

[6] A. Zheng, F. Huang, Z. Tang et al., "Stability analysis of neighborhood tunnels with large section constructed in steeply jointed rock mass," Mathematical Problems in Engineering, vol. 2020, Article ID 9641291, 14 pages, 2020.

[7] Y. Sun, J. Zuo, M. Karakus et al., "A new theoretical method to predict strata movement and surface subsidence due to inclined coal seam mining," Rock Mechanics and Rock Engineering, vol. 53, pp. 1545-1563, 2020.

[8] Z. Li, S. He, D. Song et al., "Microseismic temporal-spatial precursory characteristics and early warning method of rockburst in steeply inclined and extremely thick coal seam," Energies, vol. 14, no. 4, pp. 1-27, 2021.

[9] G. Li and Q. Yang, "Prediction of mining subsidence in shallow coal seam," Mathematical Problems in Engineering, vol. 2020, Article ID 7956947, 2020.

[10] P. Shi and Z. Gao, "The failure law of surrounding rocks overlying bed in the steep special thickness seem mining," Journal of China Coal Society, vol. 28, no. 1, pp. 13-16, 2003.

[11] D. Zhang, Comprehensive Mechanized Caving Coal Mining Stope Pressure Control, China Coal Industry Publishing House, Beijing, China, 1999.

[12] Z. Jin, Z. Hui, and S. Xuan, "Study on the motion of top-coal deformation in a fully mechanized mining field," Ground Pressure and Strata Control, vol. 1, pp. 26-31, 1992.

[13] Y. Shi, "Behaviour of rocks and mechanical model of loads on the powered supports in a fully mechanized sub-level caving face," Journal of China Coal Society, vol. 22, no. 3, pp. 253258, 1997.
[14] S. Xiao, Z. Hong, and S. Ping, "Selection of reasonable section heights during top-coal caving to steep seams," Journal of China University of Mining and Technology, vol. 38, no. 4, pp. 544-548, 2009.

[15] X. Xiong, J. Dai, and X. Chen, “Analysis of stress asymmetric distribution law of surrounding rock of roadway in inclined coal seam: a case study of shitanjing no. 2 coal seam," Advances in Civil Engineering, vol. 2020, Article ID 8877172, 14 pages, 2020.

[16] S. Wang, L. Dou, Z. Mu et al., "Study on roof breakage-induced roadway coal burst in an extrathick steeply inclined coal seam," Shock and Vibration, vol. 2019, Article ID 2969483, 14 pages, 2019.

[17] W. Yu, B. Pan, F. Zhang, S. Yao, and F. Liu, "Deformation characteristics and determination of optimum supporting time of alteration rock mass in deep mine," KSCE Journal of Civil Engineering, vol. 23, no. 11, pp. 4921-4932, 2019.

[18] F. Cui, X. Lai, and J. Cao, "Mining disturbance of horizontal section full-mechanized caving face in steeply inclined coal seam," Journal of Mining and Safety Engineering, vol. 32, no. 4, pp. 610-616, 2015.

[19] D. Chen, C. Sun, and L. Wang, "Collapse behavior and control of hard roofs in steeply inclined coal seams," Bulletin of Engineering Geology and the Environment, vol. 80, no. 2, pp. 1489-1505, 2021.

[20] Z. Liao, T. Feng, W. Yu et al., "Experimental and theoretical investigation of overburden failure law of fully mechanized work face in steep coal seam," Advances in Civil Engineering, vol. 2020, Article ID 8843172, 10 pages, 2020.

[21] G. Wu, W. Chen, S. Jia et al., "Deformation characteristics of a roadway in steeply inclined formations and its improved support," International Journal of Rock Mechanics and Mining Sciences, vol. 130, Article ID 104324, 2020.

[22] X. Qi, R. Wang, and W. Mi, "Failure characteristics and control technology of surrounding rock in deep coal seam roadway with large dip angle under the influence of weak structural plane," Advances in Civil Engineering, vol. 2020, Article ID 6623159, 17 pages, 2020.

[23] T. Hong, T. Shi, Z. Cun et al., "Characteristics of the roof behaviors and mine pressure manifestations during the mining of steep coal seam," Archives of Mining Sciences, vol. 62, no. 4, pp. 871-891, 2017.

[24] X. Li, Z. Wang, and J. Zhang, "Stability of roof structure and its control in steeply inclined coal seams," International Journal of Mining Science and Technology, vol. 27, no. 2, pp. 359-364, 2017.

[25] X. Lai, Y. Yi, S. Peng et al., "Comprehensive analysis of disaster-causing characteristics of roof stress superimposed effect in steeply inclined coal seams," Journal of China Coal Society, vol. 43, no. 1, pp. 70-78, 2018.

[26] Z. Kun, L. Xing, and W. Ning, "Test analysis for water injection technology of steep thick seam," Journal of Xi'an University of Science and Technology, vol. 30, no. 2, pp. 154158, 2010.

[27] X. Lai, P. Shan, J. Cao, H. Sun, Z. Suo, and F. Cui, "Hybrid assessment of pre-blasting weakening to horizontal section top coal caving (HSTCC) in steep and thick seams," International Journal of Mining Science and Technology, vol. 24, no. 1, pp. 31-37, 2014.

[28] G. Wang, T. Huang, S. Yan et al., "Experimental study of the fracturing-wetting effect of VES fracturing fluid for the coal seam water injection," Journal of Molecular Liquids, vol. 295, Article ID 111715, 2019. 
[29] H. Sun, X. L. Liu, S. G. Zhang et al., "Experimental investigation of acoustic emission and infrared radiation thermography of dynamic fracturing process of hard-rock pillar in extremely steep and thick coal seams," Engineering Fracture Mechanics, vol. 226, Article ID 106845, 2020.

[30] J. Lu, G. Yin, H. Gao et al., "True triaxial experimental study of disturbed compound dynamic disaster in deep underground coal mine," Rock Mechanics and Rock Engineering, vol. 53, no. 5, pp. 2347-2364, 2020.

[31] Z. Li, C. Zhang, J. Zhao et al., "Safety factor of unsaturated soil slopes considering the intermediate principal stress effect and different profiles of matric suction," Mathematical Problems in Engineering, vol. 2021, Article ID 6622522, 10 pages, 2021. 